\title{
A MUON BEAM FOR COOLING EXPERIMENTS*
}

\author{
A. Jansson, V.I. Balbekov, D. Broemmelsiek, M. Hu, N.V. Mokhov, K. Yonehara, \\ Fermilab, Batavia, IL 60510, U.S.A.
}

\begin{abstract}
Within the framework of the Fermilab Muon Collider Task Force, the possibility of developing a dedicated muon test beam for cooling experiments has been investigated. Cooling experiments can be performed in a very low intensity muon beam by tracking single particles through the cooling device. With sufficient muon intensity and large enough cooling decrement, a cooling demonstration experiment may also be performed without resolving single particle trajectories, but rather by measuring the average size and position of the beam. This allows simpler, and thus cheaper, detectors and readout electronics to be used. This paper discusses muon production using $400 \mathrm{MeV}$ protons from the Linac, decay channel and beamline design, as well as the instrumentation required for such an experiment, in particular as applied to testing the Helical Cooling Channel (HCC) proposed by Muons Inc.
\end{abstract}

\section{INTRODUCTION}

\section{Muon Cooling}

In any cooling device based on ionization there are two opposing effects. The average effect of ionization appears as a damping term in the equations of motion, thereby circumventing Liouville's theorem and allowing the phase space density to increase (under the right conditions). On the other hand, the stochastic nature of the interaction with matter, in particular multiple Coulomb scattering, generates a diffusion term, which tend to dilute the phase space. The relative size of the two terms determines the equilibrium emittance. As long as the emittance is significantly higher than the equilibrium, only the cooling (friction) term is relevant. However, any experiment to test an ionization cooling device should aim to measure and separate both effects.

\section{Beamlet Cooling Experiment}

The conventional way to test a muon cooling device is by measuring single particle phase space coordinates going into and out of the device. However, it could also be done using a macro-particle, i.e., a pencil beam or "beamlet". In particular, if a zero-emittance beam is injected into an ionization cooling device, the mapping between average input and output phase space coordinates gives the cooling term, and the increase in beam emittance gives the heating term. A potential advantage of this approach is that simpler detectors could be used, since single particles need not be resolved. By actively scanning the beam across the aperture, one can also

*Work supported by US DOE under contract DE-AC02-07CH11359 ensure that the entire phase space volume of interest is covered.

However, to perform such an experiment, one needs a very pure muon beam with a small emittance and reasonable intensity. This paper reports on the efforts to design such a beam.

\section{MuCool Test Area}

After investigating the existing Fermilab secondary beam lines and other locations such as the MiniBoone target, it was decided to further investigate the possibility of creating a muon beam at the MuCool Test Area (MTA) using $400-\mathrm{MeV}$ protons from the Linac. There were several reasons for this. The external beam lines have very limited rates. Locations, where high rates could be achieved such as after the MiniBooNE target, are in use for the foreseeable future, and would require extensive civil construction. The MTA is an area dedicated to muon cooling work, already equipped a cryo-plant, and could profit from an ample supply of Linac protons. There is a scheme by which the "leftover" protons from filling the Booster could be redirected to a production target for the MTA [1].

Since the endpoint of the muon momentum spectrum from $400-\mathrm{MeV}$ protons is only slightly above $300 \mathrm{MeV} / \mathrm{c}$, $\sim 250 \mathrm{MeV} / \mathrm{c}$ is the highest reasonable momentum for the beam line.

\section{MUON PRODUCTION}

\section{Target and Capture Solenoid}

Due to the low energy of the protons, a relatively short carbon target $(20 \mathrm{~cm})$ was found in MARS15 calculations [2] as optimal for pion production. A longer target would reduce the proton background, but also moderate the pions generated and therefore reduce the muon yield at the energies of interest. To improve the capture efficiency, the target is immersed in a $6 \mathrm{~T}$ solenoid.

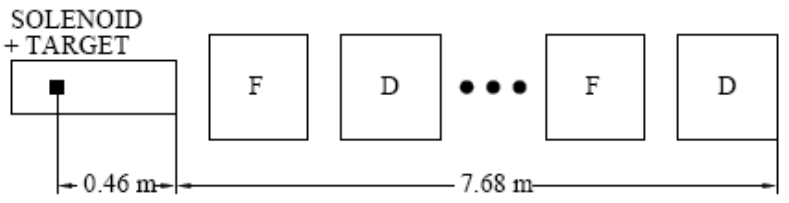

Figure 1. Schematic of the decay channel. The total number of quadrupoles is 16 .

\section{Decay Channel}

The decay channel consists of a quadrupole FODO lattice, and is a modification of an earlier design [3]. It uses BNL D2 line quads with an aperture radius of $8 \mathrm{~cm}$, a 
length of $36 \mathrm{~cm}$, and a maximum gradient of $0.1 \mathrm{~T} / \mathrm{cm} .16$ quadrupoles are used, operating at a gradient of $0.065 \mathrm{~T} / \mathrm{cm}$. A schematic is shown in Fig. 1. The muon phase space distributions at the end of the channel are given in Fig. 2, while Fig. 3 shows the muon and pion momentum spectra. The total muon yield at the end of the channel for some interesting momentum intervals are given in Table 1. The numbers were obtained using MARS15 [2] and an independent code developed specifically for this channel.
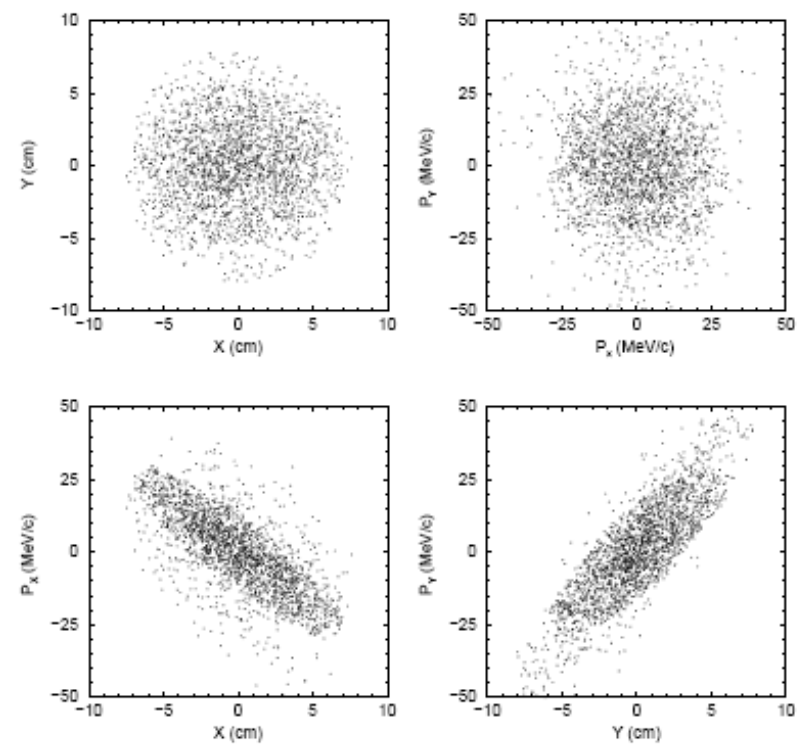

Figure 2. Muon distributions at the end of the decay channel.

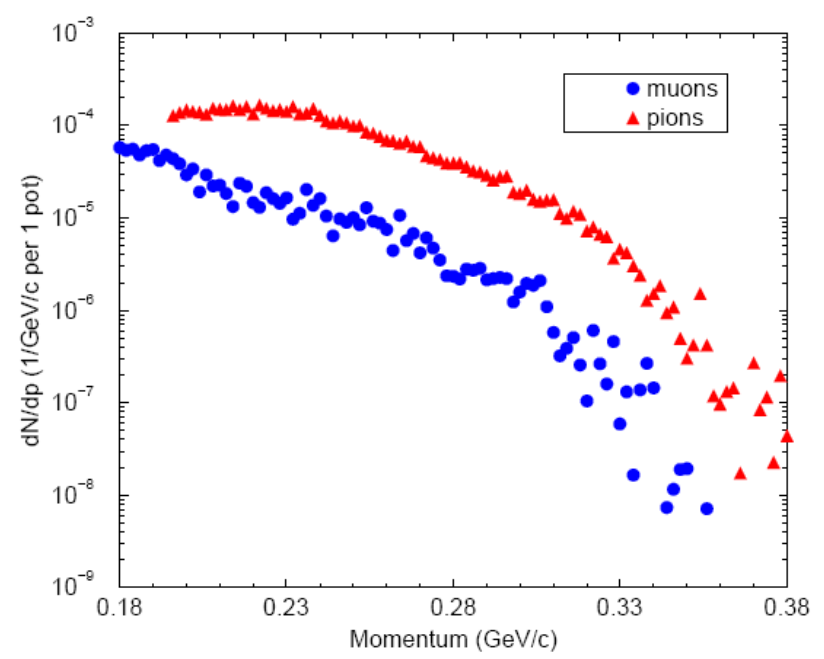

Figure 3. Muon and pion momentum spectra at the end of the decay channel.

Table 1. Number of muons at the end of the decay channel per proton on target, for three different momentum intervals.

\begin{tabular}{|l|l|l|l|}
\hline Momentum $(\mathrm{MeV} / \mathrm{c})$ & $150-200$ & $200-250$ & $250-300$ \\
\hline Muon Yield & $2.0110^{-6}$ & $1.0510^{-6}$ & $0.2110^{-6}$ \\
\hline
\end{tabular}

\section{Energy Deposition and Activation Issues}

The average energy deposition along the decay channel is $100 \mathrm{~W} / \mathrm{m}$, which is a concern. A level of around $1 \mathrm{~W} / \mathrm{m}$ is generally considered to be a reasonable limit for handson maintenance. A full energy deposition analysis has yet to be done, however.

\section{MUON BEAM LINE}

\section{Collimation and Momentum Selection}

For a beamlet cooling experiment, the muon beam should be smaller than the features that need to be resolved. Although the optimal beam size has yet to be determined, a reasonable target value is about 15-20 times smaller than the acceptance of the cooling device under test, or about $75 \mathrm{~mm} \cdot \mathrm{mrad}$ in the case of the Helical Cooling Channel. A beam of this size would also be significantly blown up by multiple scattering, thus allowing the heating term to be measured. To obtain such a small beam, the muons must be collimated to leave only the densest central part. This can be done by using stainless steel collimator blocks in the last part of the decay channel. The energy spread must also be reduced by a commensurate amount, down to a few percent. This may be achieved by using collimators in a dispersion area.

By imposing the appropriate cuts on the simulated muon distribution at the end of the decay channel, it is estimated that the muon intensity would be reduced by about a factor $10^{3}$ through the combined effect of transverse and momentum collimation. With a linac pulse of $10^{13}$ protons, this would yield about $10^{4}$ muons/pulse. Obviously, a larger emittance would yield a higher intensity. Hence, the optimal value, which will be determined by simulations, must be compromise between these two factors.

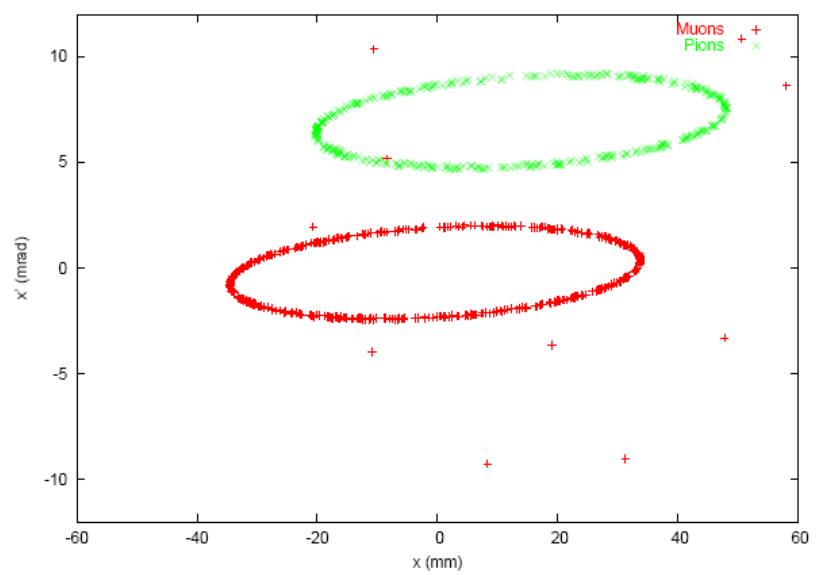

Figure 4. Muon and pion separation using a BNL "KEK style" $4.5 \mathrm{~m}$ electrostatic separator. The two ellipses representing the beams started on top of each other.

\section{Pion and Proton Separation}

Since no particle ID would be possible with a beamlet experiment, it is important that beam contamination from pions and protons is small. The decay channel and any 
other magnetic element do not distinguish between different particles with the same momentum. Therefore, the unwanted particle types must be removed by some other means. The low energy of the muons makes it undesirable to put any significant amount of material in the beam to range out the hadrons, since it would cause muons to lose energy and therefore reduce the yield of interesting muons.

An alternative way to remove contaminants is by using an electrostatic separator or Wien filter. At a given momentum, a transverse electric field gives a different kick to different particles, depending on their mass. Since the pion and muon mass are very close, this is only possible if the beam divergence is relatively small.

Electronstatic separators are available from decommissioned beamlines at Brookhaven. Fig. 4 shows the separation power of a BNL $4.5 \mathrm{~m}$ separator with a field strength of $7.5 \mathrm{MV} / \mathrm{m}$.

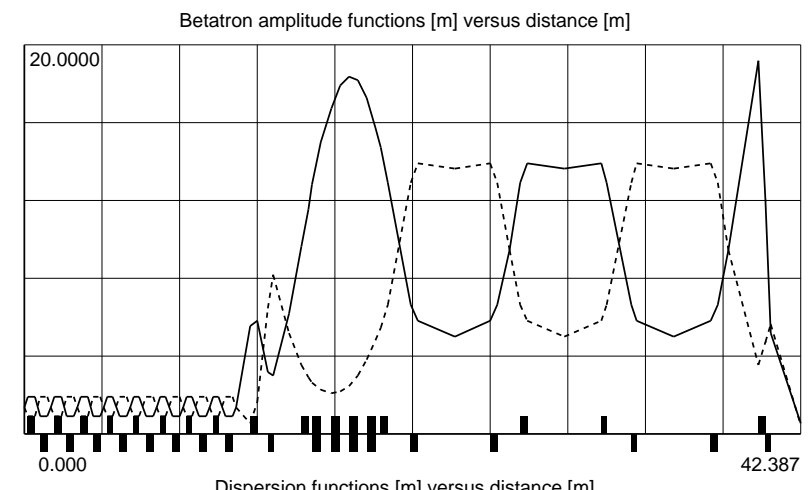

Dispersion functions $[\mathrm{m}]$ versus distance $[\mathrm{m}]$

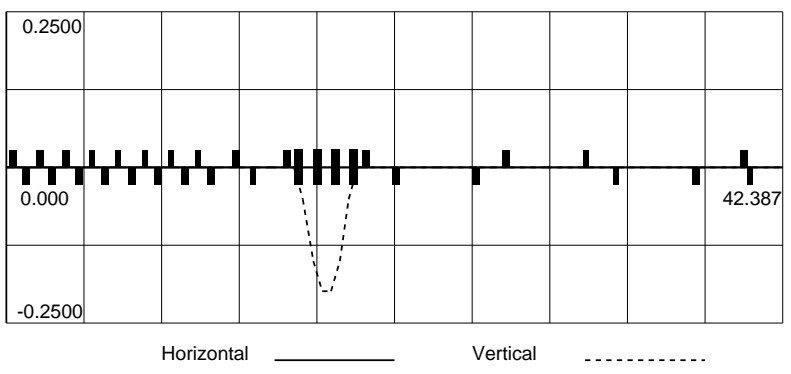

Figure 5. Preliminary optics for the muon beamline. The decay channel is matched into a section of FOFDOD lattice where beam manipulations (momentum selection, pion and proton separation) take place. Finally, the beam is matched into the device under test (e.g., the Helical Cooling Channel).

\section{Optics}

A preliminary optics design is shown in Fig. 5. The FODO lattice of the decay channel is matched by a quadrupole doublet into a section of FOFDOD lattice. This provides a series of long straight sections of alternating high- and low-beta functions. The first straight section is occupied by a momentum selection chicane. This generates vertical dispersion at a location of small vertical beta function, which provides a possibility to reduce the momentum spread using vertical collimators without significantly affecting the vertical emittance.
In the second straight section, a vertical electrostatic separator would be used to separate muons from remaining protons and pions. This is an area of high vertical beta function, which minimizes the angular spread of the beam, and therefore the size of the differential kick that needs to be given to the different species. The second straight section is used to penetrate the shield wall between the target and experimental area. The phase advance of the FOFDOD lattice is roughly 90 degrees, so the differential kick from the separator translates into a maximum differential position in the fourth straight section, where again a collimator can be used to capture the unwanted particles.

After the beam has been separated and momentum collimated, it is matched into the device under test (e.g., Helical Cooling Channel). The total length of the channel roughly corresponds to the distance available from the Linac to the beginning of the MTA experimental hall. Other optics variant, e.g., where the beamline goes all the way to the end of the hall and the turns back, doing the cooling experiment in the reverse direction, has also been discussed.

\section{Instrumentation}

In order to commission the beam line and perform any cooling experiment, the beam position and size need to be measured in multiple locations.

Scintillating fiber detectors appear to be the tool of choice, since they are very sensitive yet require little material in the beam path. A detector prototype has been developed by Fermilab PPD for the MTEST beam line [4]. This detector, which achieved single MIP sensitivity on the bench, could be adapted for use in a muon line.

When used in conjunction with bending magnets, these detectors could also be used to measure the beam momentum.

\section{SUMMARY AND OUTLOOK}

The possibility of building a $250 \mathrm{MeV} / \mathrm{c}$ muon beamline at the MuCool Test Area has been investigated. A muon yield of $210^{-6}$ in the momentum range $200-250 \mathrm{MeV} / \mathrm{c}$ was achieved at the end of the decay channel.. By collimating the muons, it is estimated that $\sim 10^{4}$ muons per linac pulse could be obtained in a small pencil beam of 75 $\mathrm{mm}$ mrad transverse emittance and a few percent momentum spread.

This is expected to be sufficient for a pencil beam cooling experiment. A full-blown simulation of the entire beamline, including collimators and electrostatic separators will be done to validate these numbers and estimate the achievable beam purity. A complete evaluation of the energy deposition and activation issues is also planned.

\section{REFERENCES}

[1] M. Popovic et al, report on protons to MTA.

[2] N.V. Mokhov, "The Mars Code System User's Guide", Fermilab-FN-628(1995), 
http://www-ap.fnal.gov/MARS/.

[3] V.I. Balbekov and N.V. Mokhov, "Low-Budget Muon Source”, PAC'01, 1553-1555, Chicago, IL (2001).
[4] H. Nguyen et al, A Fiber Profile Monitor for Low Beam Intensities, DIPAC'07, Venice, Italy. 